\title{
Planning Computer Modeled Animation: Towards an Animation Machine
}

\author{
Adailton José Alves da Cruz ${ }^{1,2}$ Alberto Barbosa Raposo ${ }^{1}$, Léo Pini Magalhães ${ }^{1}$ \\ ${ }^{1} \mathrm{DCA}$ - FEEC - UNICAMP \\ ${ }^{2}$ CEUD - UFMS \\ \{ajcruz, alberto, leopini\}@dca.fee.unicamp.br
}

\begin{abstract}
In this work the architecture of an "animation machine" is proposed. Its main goals are the definition of the animation in a more abstract level and the simulation of the characters' behaviors to detect undesired situations.
\end{abstract}

\section{Introduction}

Movement modeling techniques are described in two abstraction levels [1]. In the concrete level, movements are modeled according to their mathematical characteristics. The intentional level treats the sequence of events that causes or is affected by a movement.

An "animation machine", whose input is an animation's description in the intentional level and the output is the rendered animation, is discussed in this work. The machine also includes testing facilities to return a feedback to the animator regarding the animation's behavior.

\section{The Animation Machine}

The schema of the proposed machine is shown in Fig. 1.

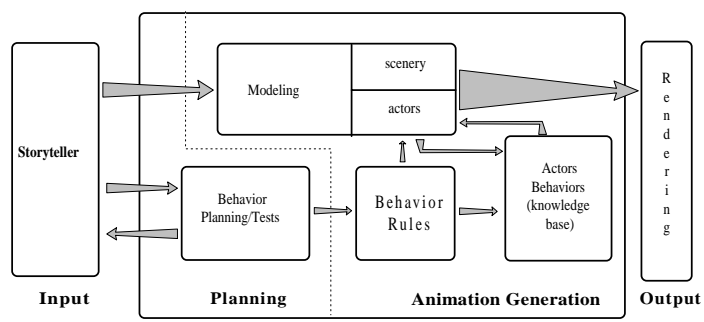

Figure 1: Architecture of the animation machine.

\subsection{Storyteller}

This module is responsible for describing the behavior associated to characters. In order to do this, the animator should describe the scene in the intentional level, like a movie director tells human actors how to act. It is necessary to investigate how techniques from artificial intelligence (AI) and visuo-linguistics can be of use in its implementation [3].

\subsection{Behavior Planning}

Behavioral characters (actors) should pass through a behavior planning module whose goal is to anticipate the animation before any frame is shot. Here actors are treated inde- pendently of their physical characteristics, considering only the interaction among them and with the animator.

An approach based on Petri Nets has proven to be a powerful way to validate the animation control model [2].

\subsection{Animation Generation}

The behavior model is passed to behavior rules module, whose function is to tell actors how they should proceed.

The actors behaviors module associates physical behaviors to actors. It is a knowledge base that knows the meaning of an actor's activity (e.g., activity "walk" for a bipedal actor is different from that of a quadrupedal one). Decisions taken by this module can be guided by AI techniques and the parameters passed by behavior rules module.

Once the physical meanings of all activities are defined, the modeling module can start the rendering process.

\section{Conclusion}

The possibilty of defining the animation in the intentional level and simulating its behavior before its actual implementation can represent a progress in the concept of computer animation. The modular approach used in the design of the animation machine also encapsulates the problems relative to each step of the process.

\section{Acknowledgements}

The first author is sponsored by CAPES/PICD and the second by FAPESP (process n. 96/06288-9).

\section{References}

[1] L. P. Magalhães. Modeling and Analysing Computer Animations. SIBGRAPI'98, 18-19.

[2] L. P. Magalhães et al. Animation Modeling with Petri Nets. Computer \& Graphics, 22(6): 735-743. 1998.

[3] S. Towns et al. Generating Coordinated Natural Language and 3D Animations for Complex Spatial Explanations. Nat. Conf. on Art. Intelligence'98, 112-119. 